\title{
Star and planet-formation with ALMA: an overview
}

\author{
Ewine F. van Dishoeck • Jes K. Jørgensen
}

Received: 13 May 2007 / Accepted: 9 July 2007 / Published online: 14 August 2007

(C) Springer Science+Business Media B.V. 2007

\begin{abstract}
Submillimeter observations with ALMA will be the essential next step in our understanding of how stars and planets form. Key projects range from detailed imaging of the collapse of pre-stellar cores and measuring the accretion rate of matter onto deeply embedded protostars, to unravelling the chemistry and dynamics of high-mass star-forming clusters and high-spatial resolution studies of protoplanetary disks down to the $1 \mathrm{AU}$ scale.
\end{abstract}

Keywords Star formation · Protoplanetary disks

\section{Introduction}

The formation of stars and planets occurs deep inside clouds and disks of gas and dust with hundreds of magnitudes of extinction, and can therefore only be studied at long wavelengths. In the standard scenario for the formation of an isolated low-mass star, a cold core contracts as magnetic and turbulent support are lost and subsequently collapses from the inside out to form a protostar with a surrounding disk. Soon after formation, a stellar wind breaks out along the rotational axis of the system and drives a bipolar outflow entraining surrounding cloud material. The outflow gradually disperses the protostellar envelope, revealing an optically visible pre-main sequence star with a disk. Inside this disk,

E.F. van Dishoeck $(\varangle)$

Leiden Observatory, P.O. Box 9513, 2300 RA Leiden,

The Netherlands

e-mail: ewine@strw.leidenuniv.nl

J.K. Jørgensen

Harvard-Smithsonian Center for Astrophysics, 60 Garden Street MS 42, Cambridge, MA 02138, USA grains collide and stick owing to the high densities, leading to pebbles, rocks and eventually planetesimals which interact to form planets. The original interstellar gas and dust is gradually lost from the disk through a combination of processes, including accretion onto the new star, formation of gas-rich planets, photoevaporation and stellar winds.

These different evolutionary stages in star- and planet formation are traditionally linked to their Spectral Energy Distributions (SEDs) (Lada 1999), which illustrate how the bulk of the luminosity shifts from far- to near-infrared wavelengths as matter moves from envelope to disk to star. So far, most tests of this scenario have been done using spatially unresolved data which encompass the entire star-disk-envelope system in a single beam. ALMA will be the first telescope capable of spatially and spectrally resolving the individual components and tracing the key physical and chemical processes on all scales.

The strengths of ALMA are (i) its high angular resolution, combined with enough sensitivity to image continuum and lines down to $0.01^{\prime \prime}(\sim 1 \mathrm{AU}=$ terrestrial planetforming zone at $150 \mathrm{pc}, 30 \mathrm{AU}=$ disk of high-mass YSO at $3 \mathrm{kpc}$ ); (ii) its high spectral resolution down to $0.01 \mathrm{~km} \mathrm{~s}^{-1}$ so that the details of the dynamics and kinematics can be probed; (iii) access to thousands of lines from hundreds of species allowing a wide variety of physical and chemical regimes to be probed; and (iv) its ability to detect optically thin dust emission and thus directly derive dust masses.

ALMA probes the wavelength range of 0.3-9 millimeter, which is on the Rayleigh-Jeans tail of the SEDs of young stellar objects. For a complete picture of these sources complementary space and ground-based observations at shorter wavelengths are necessary. Compared with other missions, ALMA is less well suited for large area surveys because of its small field of view. Mid-infrared observatories such as the Spitzer Space Telescope at 3-70 $\mu \mathrm{m}$ probe the peak 
of the SED for low-mass YSOs and can scan large areas much more rapidly, albeit at lower spatial and spectral resolutions. Near-infrared imaging is a powerful tool to characterize the stellar component, whereas the Herschel Space Observatory and ground-based single-dish submillimeter telescopes equipped with large format bolometers can rapidly search large areas for cold dust emission. These missions will provide complete unbiased catalogs with thousands of sources covering all the nearby molecular clouds and star-forming regions within a few hundred pc (contained within Gould's Belt), many of the young clusters and associations within $1 \mathrm{kpc}$, and most of the prominent high-mass star-forming clouds to the outer edge of the Galaxy. Thus, the primary source lists for ALMA will come from these missions. They will also provide the main statistical results from which, for example, timescales for the different phases can be derived.

This paper outlines a number of key questions for each evolutionary state where ALMA can make a major contribution. It focusses mostly on low-mass star formation and protoplanetary disks, but many of the same arguments are also valid for high-mass star formation. Inspiration for this review was provided by the many beautiful paintings by Juan Miró displayed in Madrid and elsewhere around the world. Most appropriate for this topic are 'Birth of the world', 'Chiffres and constellations', 'Red disk' and 'Serpent looking at comet'. A challenge for the reader is to find the relations between these paintings and the topics described here.

\section{Low-mass star formation}

\subsection{Pre-stellar cores}

Question 1 What are the initial conditions for low-mass star formation, in particular the physical structure and kinematics of the densest part of the core?

In recent years, a number of cold, highly extincted clouds have been identified which have a clear central density condensation. These so-called pre-stellar cores are believed to be on the verge of collapse and thus represent the earliest stage in the star-formation process (e.g., Tafalla et al. 1998). The physical and chemical state of these clouds is now well established on scales of few thousand AU by single dish millimeter observations combined with extinction maps. The cores are cold, with temperatures varying from $10-15 \mathrm{~K}$ at the edge to as low as 7-8 $\mathrm{K}$ at the center, and have density profiles that are well described by Bonnor-Ebert profiles. It is now widely accepted that most molecules are highly depleted in the inner denser parts of these cores (Caselli et al. 1999; Bergin et al. 2002): images of clouds such as B68 show only a ring of $\mathrm{C}^{18} \mathrm{O}$ emission, with more than $90 \%$ frozen out toward its center.
ALMA will be particularly powerful in probing the central part of the core on scales of $100 \mathrm{AU}$ and search for signs of collapse in the very earliest stages. Important probes are the lines of $\mathrm{N}_{2} \mathrm{H}^{+}$and $\mathrm{H}_{2} \mathrm{D}^{+}$at $372 \mathrm{GHz}$, with the latter line a unique probe of the kinematics in regions where all heavy molecules are depleted (van der Tak et al. 2005).

\subsection{Very low luminosity objects and formation of brown dwarfs}

Question 2 What prevents some clouds from collapsing? Why do some low-luminosity sources have such a low accretion rate in spite of the much larger reservoir of gas and dust?

About $75 \%$ of so-called 'starless' cores (i.e., dark cores with no IRAS source) remain starless down to $0.01 \mathrm{~L}_{\odot}$ or less even after deep surveys with Spitzer (Kirk et al. 2007). However, Spitzer has revealed a small set of cores with so-called Very Low Luminosity Objects (VeLLOs). Examples include L1014 $\left(\sim 0.1 \mathrm{~L}_{\odot}\right.$, Young et al. 2004$), \mathrm{L} 1521 \mathrm{~F}$ $\left(\sim 0.05 \mathrm{~L}_{\odot}\right.$, Crapsi et al. 2005; Bourke et al. 2006), and IRAM $04191\left(\sim 0.08 \mathrm{~L}_{\odot}\right.$, Dunham et al. 2006). These VeLLOs are embedded in cores with typical masses of $1 \mathrm{M}_{\odot}$, but their low luminosities suggest that their central stellar masses are low and that they (currently) have low accretion rates. They also show very different outflow properties ranging from a large well developed outflow in IRAM 04191 to a miniscule outflow in L1014 only detectable through high angular resolution millimeter observations (Fig. 1). This suggests that accretion in these cores may be episodic. It remains an interesting question whether these VeLLOs constitute a separate stage in the evolution of low-mass protostars or are precursors of substellar objects, but without a better handle on their dynamical structure it is difficult to predict the "end result" of the ongoing star formation in these cores. ALMA will be able to zoom in on these sources, image their disks (whose presence is inferred from the SEDs) and small scale outflows and furthermore constrain the kinematics of their envelopes.

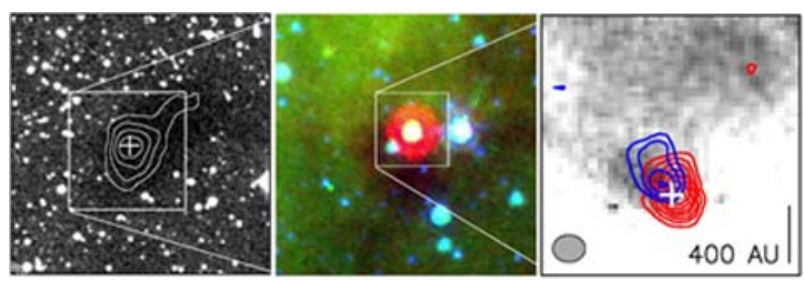

Fig. 1 The VeLLO L1014-IRS. Left: optical image with $1.2 \mathrm{~mm}$ dust continuum emission overlayed. Middle: Spitzer mid-infrared image with $4.5 \mu \mathrm{m}$ (blue), $8.0 \mu \mathrm{m}$ (green) and $24 \mu \mathrm{m}$ (red). Right: CO 2-1 map of the innermost region of the core from the SubMillimeter Array (SMA). Images from Young et al. (2004) (left, middle) and Bourke et al. (2005) (right) 
Question 3 How do brown dwarfs form? Like stars or like planets?

The VeLLOs described above could be the precursors of substellar objects like brown dwarfs. If so, the presence of outflows and accretion disks imply a formation process similar to that of stars. Formation in, or fragmentation of, a disk around a more massive primary star is not consistent with these data. Many other lines of evidence based on infrared imaging and spectroscopy point in the same direction (e.g., Testi et al. 2002; Mohanty et al. 2004). Large samples of young brown dwarfs now exist, several of them in wide binaries which are easily disrupted, providing further clues on their origin (see for a review Luhman et al. 2007). ALMA will be able to determine whether brown dwarfs in the earliest stages have similar statistics in terms of binary fraction and separation.

\subsection{Formation of stellar clusters and origin of the IMF}

Question 4 What is the relation between cloud or clump structure and the IMF?

The advent of large bolometer arrays on submillimeter telescopes has revived detailed studies of the structure of molecular clouds and cores just prior to and during the star formation process. On large pc-size scales, molecular clouds have a highly inhomogeneous or 'clumpy' structure, from which the mass distribution $\Delta N / \Delta M$ can be measured (see Williams et al. 2000, for a review). Interestingly, the mass spectrum of cores follows a law $\propto M^{-1.5}$ below $0.5 \mathrm{M}_{\odot}$ and $\propto M^{-2.5}$ above $0.5 \mathrm{M}_{\odot}$ (Alves et al. 2007), similar to the stellar initial mass function (IMF). This suggests that the IMF may already be determined at the pre-stellar stage during the fragmentation of a (turbulent) molecular cloud, a result with wide-ranging implications for studying the evolution of molecular clouds in our Galaxy and galaxies as a whole. The sensitivity and spatial resolution of ALMA are needed, however, to separate the lower density cloud material from the dense cores and to link this work with the optical and infrared determinations of the low-mass end of the IMF in young clusters. For example, the mass spectrum is still uncertain for clump masses below $0.1 \mathrm{M}_{\odot}$, whereas ALMA can probe the mass function down to planetary masses and study the origin and distribution of brown dwarfs and freefloating Jupiter-mass exo-planets using also kinematic information.

Question 5 What fraction of stars forms as binaries or multiples? Are all cluster members co-eval? Is there evidence for dynamical interactions?
Related to Question 4 is the question whether cloud fragmentation leads to a young cluster or to distributed star formation. In contrast with previous claims, Spitzer finds evidence for both processes, including a significant fraction of young stars distributed throughout the clouds (e.g., Allen et al. 2007). The formation of the more massive members of a cluster through competitive accretion is also heavily debated (e.g., Bate and Bonnell 2005; Krumholz et al. 2005). ALMA can address this question by determining masses of YSOs in the earliest, deeply embedded stages when accretion is still taking place. The fraction of binaries and multiples can be compared with those of optically visible $\mathrm{T}$ Tauri stars and field main-sequence stars, providing clues on binary evolution and dynamical interactions (e.g., ejection).

Spitzer data show a wide variety of SEDs of clusters of YSOs on 0.1 pc scales (e.g., Rebull et al. 2007; Jørgensen et al. 2006). What causes this diversity? The normal assumption is that the different SEDs (rising, falling) reflect a real age spread from $<0.1$ to $>1 \mathrm{Myr}$ as part of a more-or-less uniform star formation process. However, on scales as small as $0.1 \mathrm{pc}$ it is also reasonable to assume that all objects form quasi-simultaneously from a fragmenting core. In such a coeval scenario, the diversity in SEDs would imply that objects go through the evolutionary states at different rates. ALMA and mid-infrared data will be needed to settle this.

\subsection{Embedded YSOs: infall vs. outflow}

Question 6 What are the accretion rates during the earliest stages of star formation and how do they vary with time?

Deeply embedded young stellar objects (the so-called 'Class 0' objects) have a complex physical and kinematical structure, with envelopes, disks and outflows all blurred together in current single-dish observations. High spatial and spectral resolution ALMA data will be essential to disentangle the infall, outflow and rotation components of these systems and study their evolution. Of particular importance will be to measure directly the accretion rates onto the disk and star. Redshifted absorption against the continuum is thought to be the most direct tracer (Fig. 2) but this absorption is completely overwhelmed by large-scale emission in singledish data. Interferometer data reveal such red-shifted absorption in a few (but not all) Class 0 sources but only in low-density tracers perhaps indicative of large-scale infall rather than small-scale accretion (e.g., Jørgensen et al. 2007; Di Francesco et al. 2001). ALMA will have orders of magnitude higher sensitivity to infall tracers due to the combination of larger collecting area and smaller beam.

Question 7 What drives outflows and how does the outflow structure change with time? 


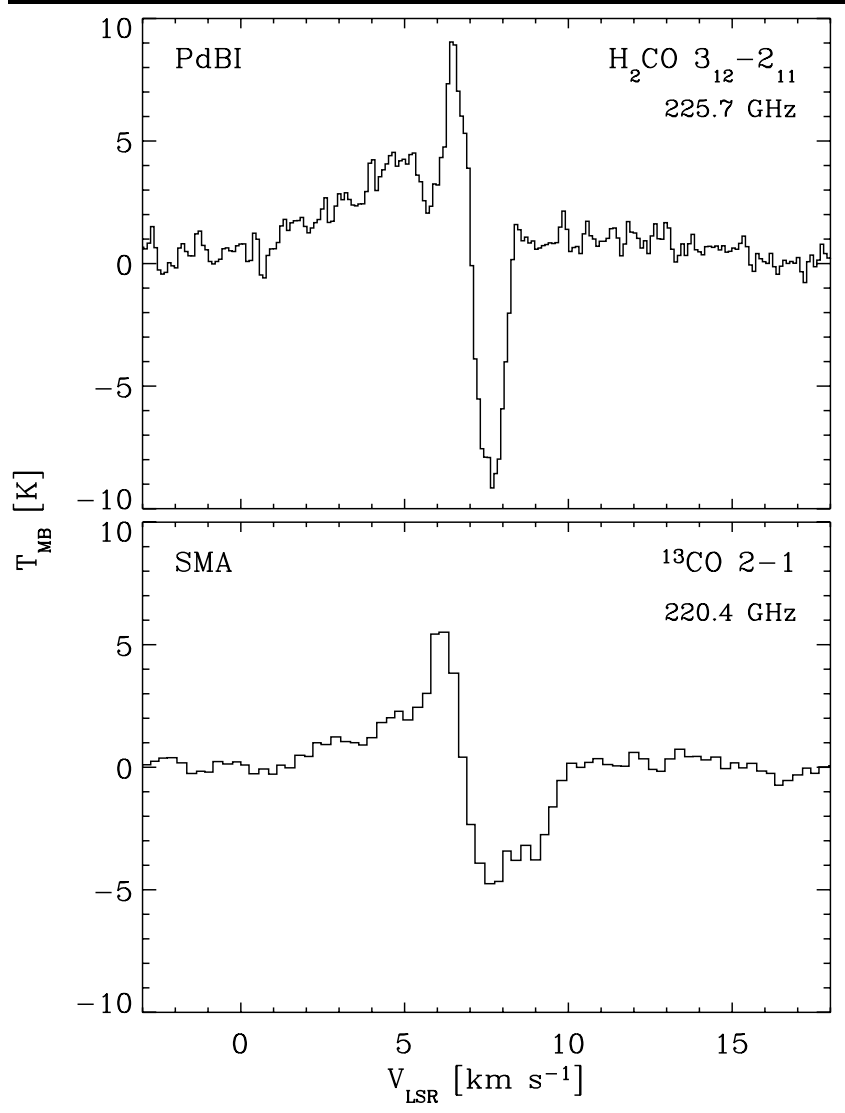

Fig. 2 Inverse $\mathrm{P}$ Cygni profiles toward the Class 0 YSO NGC1333-IRAS4A in lines of $\mathrm{H}_{2} \mathrm{CO}$ from IRAM Plateau de Bure observations (Di Francesco et al. 2001) and ${ }^{13} \mathrm{CO}$ from SMA observations (Jørgensen et al. 2007)

Violent outflows are a key characteristic of star formation (Richer et al. 2000). Although they have been studied with single-dish telescopes for more than 25 years, the mechanism of their formation remains poorly understood (Shang et al. 2007). In the most deeply embedded objects, highly collimated jet-like molecular outflows with extreme velocities up to $200 \mathrm{~km} \mathrm{~s}^{-1}$ are observed, but when the protostar evolves, both the mechanical power and the collimation seem to decrease, suggesting that the former is due to a decline in the overall mass accretion rate (e.g., Bachiller and Tafalla 1999; Arce and Sargent 2006) (Fig. 3). ALMA will permit studies of many different kinds of YSOs, and, combined with independent estimates of the mass accretion rate (see above), test magnetohydrodynamical models of their evolution. The actual location and mechanism by which outflows are launched, and whether they are episodic in nature, is still a subject of intense debate. ALMA will provide detailed images of the disk/outflow interface down to a few AU scales where the outflow is accelerated and where the most intense interactions between the outflow and its surroundings take place.
Question 8 What is the role of outflows in determining the final mass of a star and in dispersing the core?

Comparisons between cloud core and stellar mass functions show great similarity in shape (see Sect. 2.3) but with a shift in mass of typically a factor of $\sim 3$ (Alves et al. 2007). Is this largely due to the action of outflows dispersing the cores? Also, what is the role of outflows in carrying off angular momentum? Attempts to measure the mass dispersion rate in embedded YSOs have traditionally suffered from poor spatial resolution. Interferometer data are needed to measure the local specific angular momentum as a function of radius in the protostellar envelope, from the largescale core to the rotationally supported disk-like structures (e.g., Hogerheijde 2001; Takahashi et al. 2006).

\section{High-mass star formation}

Question 9 Is high-mass star formation a scaled-up version of low-mass star formation? What triggers high-mass star formation?

High-mass stars $\left(\gtrsim 10 \mathrm{M}_{\odot}, \gtrsim 10^{4} \mathrm{~L}_{\odot}\right)$ play a major role in the interstellar energy budget and the shaping of the Galactic environment (e.g., Cesaroni 2005). Phenomena associated with massive stars such as photoionization, powerful winds, shocks, expanding H II regions and supernovae drastically modify the interstellar medium. Due to large distances, short time scales, and heavy extinction, the formation of high mass stars is still poorly understood compared to that of their lower mass counterparts. The earliest stages of massive star formation have been revealed as dark clouds seen in absorption against mid-infrared emission (the so-called 'Infrared Dark Clouds') (e.g., Simon et al. 2006) and systematic surveys are attempting to put the various observational signposts in an evolutionary sequence starting with centrally condensed clouds with masers (so-called High-Mass Protostellar Objects), followed by hot cores with high temperature ( $>100 \mathrm{~K}$ ) and high abundances complex organic molecules (Hot Molecular Cores), and subsequently ultra-compact $\mathrm{H}$ II regions showing significant amounts of ionized gas.

The similarity of some of these stages with those of their lower-mass counterparts, coupled with the detection of outflows and signs of rotating disks (e.g., Shepherd and Churchwell 1996; Shepherd and Kurtz 1999), suggest a common formation mechanism for all stars. However, while these similarities may hold for young B-type stars, the situation is much less clear for O-type stars and the debate between the turbulent cloud fragmentation scenario and that of competetive accretion or mergers is still far from settled (McKee and Tan 2003; Krumholz et al. 2005; Bonnell and Bate 2006). A related question is whether the formation of massive stars 


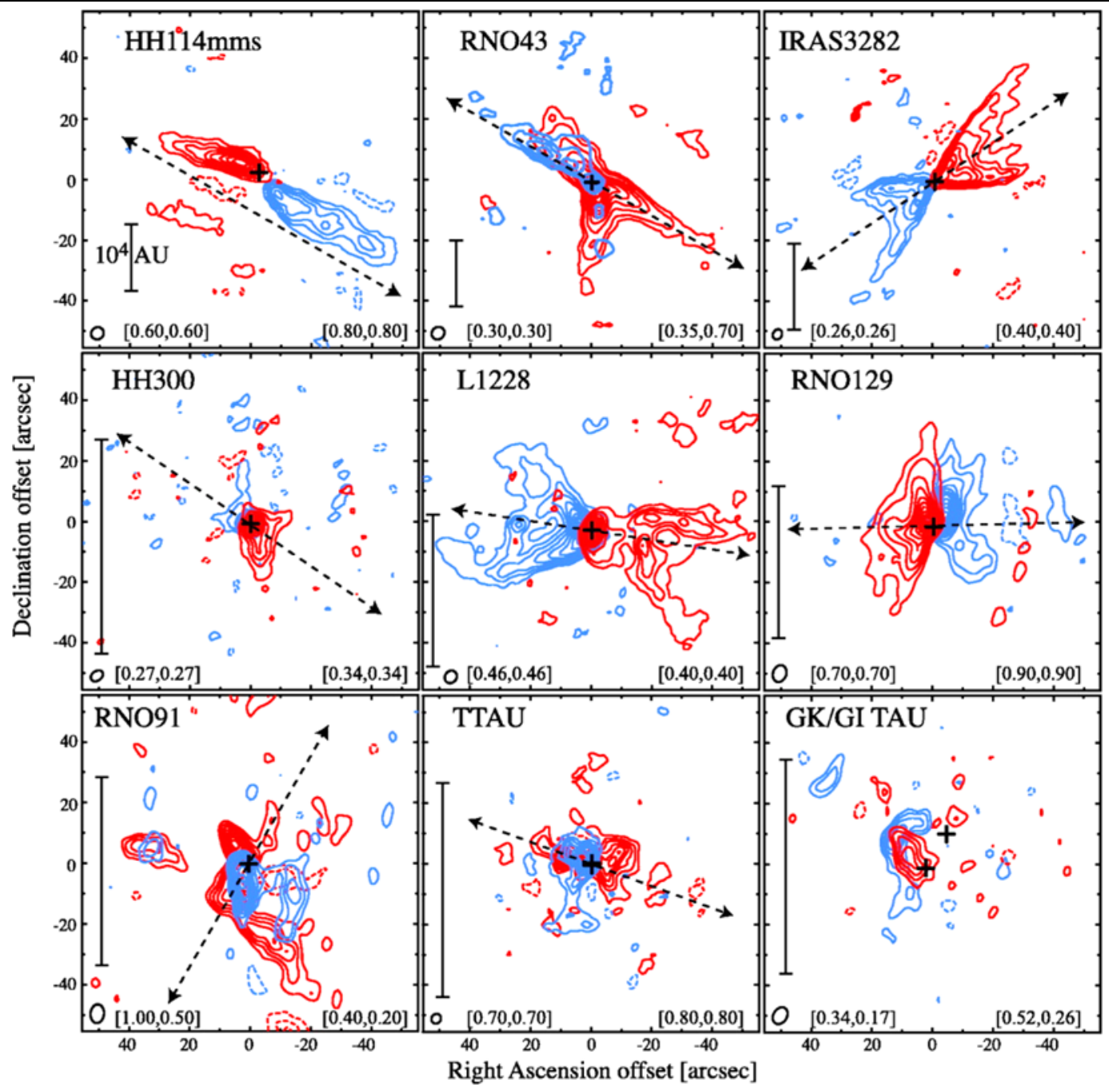

Fig. 3 Gallery of ${ }^{12} \mathrm{CO} 1-0$ outflows from the OVRO survey of Arce and Sargent (2006) with Class 0 objects in the top, Class I in the middle and Class II in the bottom panels. In each panel the cross mark the position of the protostar given by millimeter continuum observations

is largely triggered, either by shocks compressing the cloud material ('collect and collapse') or by 'radiation-driven implosion'. Clear observational evidence for triggered star formation is still lacking. Examination of cluster properties and separating the more diffuse clump and denser core material will be particularly relevant to determine which process dominates in which environment. Contrasting star formation in the outer Galaxy, where metallicity, densities, radiation field and gravitational potential well are lower compared with the inner Galaxy may also be revealing.

\section{Astrochemistry}

Question 10 Which molecule is best suited to trace which physical component of the YSO environment? Can we use chemistry as a 'clock' of the evolutionary state of the object?
Systematic studies of molecules in YSOs are starting to reveal the different chemical characteristics associated with the various stages star formation (e.g., Jørgensen et al. 2004; Ceccarelli et al. 2007; van Dishoeck 2006). The coldest pre-stellar cores show heavy freeze-out of virtually all gasphase molecules onto the cold grains (see Sect. 2.1), where grain-surface chemistry can lead to more complex species. Once the protostar starts to heat the envelope, the ices will evaporate in a sequence according to their sublimation temperatures, with the most volatile species like $\mathrm{CO}$ coming off at temperatures as low as $20 \mathrm{~K}$ and the most strongly bound species like $\mathrm{H}_{2} \mathrm{O}$ around $100 \mathrm{~K}$. The impact of outflows on the inner envelope can also liberate molecules from the ices and sputter grain cores. The evaporated ices subsequently drive a rapid gas-phase chemistry for a period of $10^{4}-10^{5}$ yr. For example, reactions with evaporated $\mathrm{CH}_{3} \mathrm{OH}$ are thought to lead to high abundances of $\mathrm{CH}_{3} \mathrm{OCH}_{3}$ and $\mathrm{HCOOCH}_{3}$, although a grain surface origin of these mole- 
cules is also possible (e.g., Bisschop et al. 2007; Bottinelli et al. 2007). These so-called 'hot cores' are signposts of the earliest stages of high-mass star formation, and are now also found around some low-mass YSOs. After $\sim 10^{5} \mathrm{yr}$, the abundances are reset by ion-molecule chemistry to their normal cloud values, leading to the potential of molecules to act as chemical clocks.

So far, this scenario is almost entirely based on spatially unresolved single-dish data, with smaller-scale structure extracted from observations of multiple lines of the same molecule with different excitation conditions. Limited interferometer data confirm the different chemical zones (e.g., Jørgensen 2004), but only ALMA will have the combined sensitivity, spatial resolution and $(u, v)$ coverage to make chemical 'images' of YSOs in large sets of lines necessary to directly test chemical models and explore and develop the use of molecules as clocks of star formation.

Question 11 How far does chemical complexity go? Can we find (the building blocks of) pre-biotic molecules?

Of the $>130$ different molecules detected in interstellar clouds, the majority $(\sim 75 \%)$ are organics, including species as complex as ethyl-cyanide $\left(\mathrm{C}_{2} \mathrm{H}_{5} \mathrm{CN}\right)$, acetamide $\left(\mathrm{CH}_{3} \mathrm{CONH}_{2}\right.$, the largest interstellar molecule with a peptide bond) and glycol-aldehyde $\left(\mathrm{CH}_{2} \mathrm{OHCHO}\right.$, the first interstellar sugar) (e.g., Hollis et al. 2000, 2006). However, in spite of literature claims, the simplest amino-acid glycine $\left(\mathrm{NH}_{2} \mathrm{CH}_{2} \mathrm{COOH}\right)$ has not yet been convincingly detected. Current instrumentation prevents deep searches for more complex molecules for several reasons: (a) the regions of high chemical complexity are often very small $\left(<1^{\prime \prime}\right)$, the typical sizes of hot cores; (b) the crowding of lines is usually so high that the confusion limit is reached; and (c) the largest molecules have many close-lying energy levels so that the intensity is spread over many different lines, each of them too weak to detect. ALMA will be able to push the searches for prebiotic molecules two orders of magnitude deeper to abundances of $<10^{-13}$ with respect to $\mathrm{H}_{2}$, because it will have a much higher sensitivity to compact emission and will resolve the sources so that spatial information can be used to aid identifications of lines.

Question 12 Which fraction of complex molecules will end up unaltered in the protoplanetary disk? How are they modified before incorporation into planetary systems?

The dynamics of gas in the inner few hundred AU of protostellar envelopes are not yet well understood, but are important to determine whether some of the observed (complex) molecules end up in the rotating disk. Also, the disk entry point is highly relevant, since gas falling in too close to the star will experience such a strong accretion shock onto the disk that all molecules will dissociate (Neufeld and Hollenbach 1994) and only molecules entering at much larger distances survive. ALMA's high spatial and kinematic resolution will obviously be needed to address this issue.

Once in the disk, the chemistry is governed by similar gas-phase and gas-grain interactions as in envelopes, but at higher densities. Also, UV and X-rays from the young star dissociate molecules and modify the chemistry in the optically thin surface layers. This results in a layered chemical structure, with a top layer consisting mostly of atoms, a mid-plane layer where most molecules are frozen out, and an intermediate layer where the dust grains are warm enough to prevent complete freeze-out and where molecules are sufficiently shielded from radiation to survive (for a review, see Bergin et al. 2007). So far, chemical images have been limited to just a few pixels across a handful of disks in a few lines (e.g., Qi et al. 2003; Piétu et al. 2007). Obviously, ALMA will throw this field wide open. A particularly exciting topic is the chemistry in the inner disk, i.e., inside the 'snow-line' where all molecules evaporate and the chemistry approaches that at LTE. For example, Spitzer data have revealed highly abundant and hot HCN in the inner disk (Lahuis et al. 2006). The brightness temperatures of the submillimeter lines are predicted to be several hundred K, sufficient for ALMA to image the inner few $\mathrm{AU}$ in the nearest disks.

\section{Protoplanetary disks}

\subsection{Young disks in the embedded phase}

Question 13 How do disks form and grow with time? How hot or cold is the disk? Can we find evidence for gravitational instabilities?

The study of disk formation in the earliest stages will be a central scientific goal of ALMA. Key questions include whether most of the disk mass is already assembled in the earliest Class 0 phase (e.g., Fig. 4), or whether disk growth - and thus stellar growth - continues in the later stages. Also, the dynamics of disk formation and how this depends on initial core parameters (e.g., core rotation) are unclear. Through high spatial resolution kinematic data, ALMA can image the Keplerian motions of the gas and thus obtain direct estimates of stellar mass. Tracing $M_{\text {env }} / M_{\text {disk }}$ and $M_{\text {disk }} / M_{*}$ for a wide variety of stellar types as a function of evolution and testing scenarios such as that shown in Fig. 5 will be a major legacy of ALMA. Multi-line observations can determine the gas temperature and the amount of heating through accretion shocks vs. UV radiation. Finally, images of young disks can reveal asymmetries or spiral arms (e.g., Lin et al. 2006), indicative of gravitational instabilities which may lead to giant planet formation (Boss 2003). 


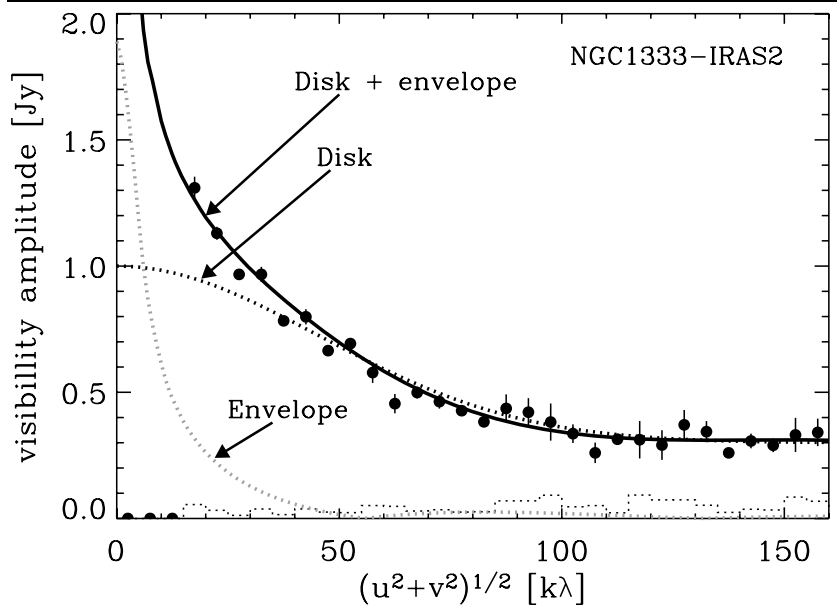

Fig. 4 Interferometric $850 \mu \mathrm{m}$ continuum observations of the NGC 1333-IRAS2A Class 0 YSO from the SMA (Jørgensen et al. 2005). The data show the presence of a compact source of emission not accounted for by the larger scale envelope models. Rather, the data can be well-fit by a combination of the extended envelope and a $300 \mathrm{AU}$ circumstellar disk

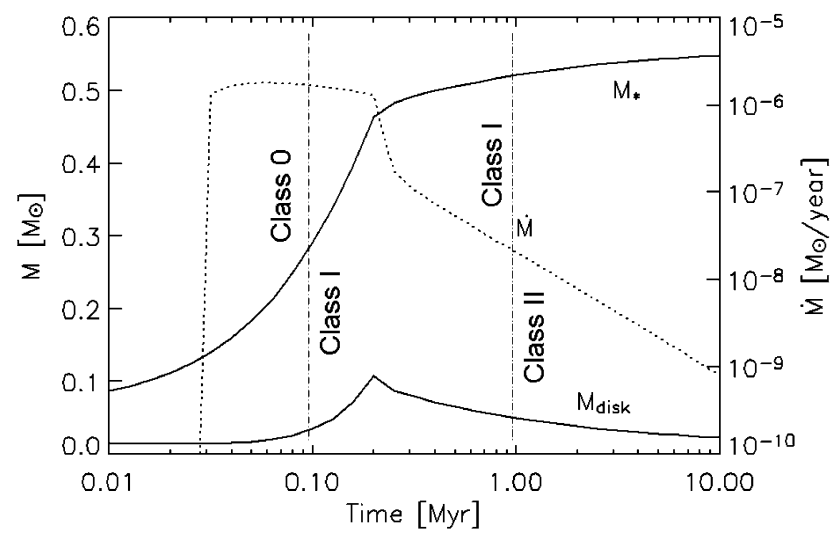

Fig. 5 Model for the evolution of disk and stellar masses (solid lines) and accretion rate from the disk onto the star from the Class 0 through Class II stages of young stellar objects. Figure from Dullemond et al. (2007) after Hueso and Guillot (2005)

\subsection{Disks around pre-main sequence stars}

Question 14 What is the physical and chemical structure of the inner planet-forming zones of disks?

Large gas disks with masses of $\sim 10^{-2} \mathrm{M}_{\odot}$ have been revealed around classical $\mathrm{T}$ Tauri and Herbig Ae stars with ages of a few Myr by millimeter continuum and CO line emission, providing constraints on masses and sizes of disks, their velocity patterns and even the level of turbulence (e.g., Koerner and Sargent 1995; Dutrey et al. 1996; Andrews and Williams 2007; Qi et al. 2003). However, these data only probe the outer ( $>50 \mathrm{AU}$ ) disk region. ALMA will have the sensitivity to detect and image all the dust in the disk down to the $1 \mathrm{AU}$ scale. Through multifrequency ob- servations, ALMA will also be able to measure the change of dust properties within disks, perhaps showing direct evidence for radius-dependent grain growth in the midplane, up to sizes of several mm. ALMA will have the sensitivity to map optically thick lines at a few AU resolution, providing information about the gas content and its chemistry and kinematics down to the planet-forming zones. The stellar masses inferred from kinematics form important direct tests of pre-main-sequence stellar evolution models. By imaging lines with different excitation conditions, maps of the $\mathrm{H}_{2}$ density distribution will become possible. Together, such ALMA data can provide unbiased surveys of disks in different star-forming regions, down to an equivalent sensitivity of a few Earth masses of dust and gas, and probe the distribution of disk parameters with stellar mass, luminosity, age and environment.

\subsection{Disk evolution and gap formation}

Question 15 When and how do gas and dust disappear from the disks? Do they disappear at the same time? Are there multiple paths from gas-rich disks to the debris disk stage?

Near-infrared surveys have shown that inner dust disks $(<$ few AU) disappear on timescales of a few Myr (Haisch et al. 2001). Surveys with Spitzer at mid-infrared wavelengths are starting to reveal a similar trend for the planetforming zones out to $\sim 10 \mathrm{AU}$, with a significantly lower disk fraction for weak-line $\mathrm{T}$ Tauri stars than for their classical counterparts (Cieza et al. 2007). Indeed, examination of hundreds of SEDs of stars with disks in Spitzer surveys show that there may be multiple evolutionary paths from the massive gas-rich disks to the tenuous gas-poor debris disks, involving both grain growth and gap opening. ALMA will be critical to study these transitional objects by imaging the holes or gaps in their dust disks down to a few AU and measure the remaining gas mass through tracers like $\mathrm{CO}$ and [C I], some of which may be left inside the holes. Giant planet formation, grain growth and photoevaporation are the three major contending theories for explaining holes in the dust disks but they have different predictions for the gas vs. large dust distribution. Overall, surveys for gaps in disks can provide statistics on the frequency and timescale for planet formation.

\section{Conclusions}

ALMA will be vital and unique to answer key questions in star- and planet formation, by resolving the physical processes taking place during the collapse of molecular clouds, imaging the structure of protostars and of protoplanetary disks, and determining the chemical composition 
of the material from which future solar systems are made. Many of the ALMA source lists will come from unbiased surveys being carried out now, in particular Spitzer, Herschel, near-infrared and single-dish submillimeter surveys. To extract information from ALMA data, however, sophisticated analysis and modeling tools are needed. The community needs to invest now in those tools to ensure that they are ready by the time that ALMA is fully commissioned.

Other major facilities in the timeframe of ALMA operations include the James Webb Space Telescope and groundbased extremely large optical telescopes (ELTs). These facilities will be highly complementary to ALMA, each addressing a different part of the star- and planet formation puzzle. There is no doubt, however, that ALMA will be the key instrument for much of the physics and chemistry associated with star- and planet formation.

\section{References}

Allen, L., Megeath, S.T., Gutermuth, R., et al.: In: Reipurth, B., Jewitt, D., Keil, K. (eds.) Protostars and Planets V, pp. 361-376. University of Arizona Press, Tucson (2007)

Alves, J., Lombardi, M., Lada, C.J.: Astron. Astrophys. 462, L17 (2007)

Andrews, S.M., Williams, J.P.: Astrophys. J. 659, 705 (2007)

Arce, H.G., Sargent, A.I.: Astrophys. J. 646, 1070 (2006)

Bachiller, R., Tafalla, M.: In: Lada, C.J., Kylafis, N.D. (eds.) The Origin of Stars and Planetary Systems, p. 227. Kluwer Academic, Dordrecht (1999)

Bate, M.R., Bonnell, I.A.: Mon. Not. Roy. Astron. Soc. 356, 1201 (2005)

Bergin, E.A., Alves, J., Huard, T., Lada, C.J.: Astrophys. J. 570, L101 (2002)

Bergin, E.A., Aikawa, Y., Blake, G.A., van Dishoeck, E.F.: In: Reipurth, B., Jewitt, D., Keil, K. (eds.) Protostars and Planets V, pp. 751-766. University of Arizona Press, Tucson (2007)

Bisschop, S.E., Jørgensen, J.K., van Dishoeck, E.F., de Wachter, E.B.M.: Astron. Astrophys. 465, 913 (2007)

Bonnell, I.A., Bate, M.R.: Mon. Not. Roy. Astron. Soc. 370, 488 (2006)

Boss, A.P.: Astrophys. J. 599, 577 (2003)

Bottinelli, S., Ceccarelli, C., Williams, J.P., Lefloch, B.: Astron. Astrophys. 463, 601 (2007)

Bourke, T.L., Crapsi, A., Myers, P.C., et al.: Astrophys. J. 633, L129 (2005)

Bourke, T.L., Myers, P.C., Evans, N.J., et al.: Astrophys. J. (2006, in press)

Caselli, P., Walmsley, C.M., Tafalla, M., Dore, L., Myers, P.C.: Astrophys. J. 523, L165 (1999)

Ceccarelli, C., Caselli, P., Herbst, E., Tielens, A.G.G.M., Caux, E.: In: Reipurth, B., Jewitt, D., Keil, K. (eds.) Protostars and Planets V, pp. 47-62. University of Arizona Press, Tucson (2007)

Cesaroni, R.: Astrophys. Space Sci. 295, 5 (2005)

Cieza, L., Padgett, D.L., Stapelfeldt, K.R., et al.: Astrophys. J. (2007, in press)

Crapsi, A., Caselli, P., Walmsley, C.M., et al.: Astrophys. J. 619, 379 (2005)

Di Francesco, J., Myers, P.C., Wilner, D.J., Ohashi, N., Mardones, D.: Astrophys. J. 562, 770 (2001)

Dullemond, C.P., Hollenbach, D., Kamp, I., D’Alessio, P.: In: Reipurth, B., Jewitt, D., Keil, K. (eds.) Protostars and Planets V, pp. 555572. University of Arizona Press, Tucson (2007)

Dunham, M.M., Evans, N.J. II, Bourke, T.L., et al.: Astrophys. J. 651, 945 (2006)

Springer
Dutrey, A., Guilloteau, S., Duvert, G., et al.: Astron. Astrophys. 309, 493 (1996)

Haisch, Jr. K.E., Lada, E.A., Lada, C.J.: Astrophys. J. 553, L153 (2001)

Hogerheijde, M.R.: Astrophys. J. 553, 618 (2001)

Hollis, J.M., Lovas, F.J., Jewell, P.R.: Astrophys. J. 540, L107 (2000)

Hollis, J.M., Lovas, F.J., Remijan, A.J., et al.: Astrophys. J. 643, L25 (2006)

Hueso, R., Guillot, T.: Astron. Astrophys. 442, 703 (2005)

Jørgensen, J.K.: Astron. Astrophys. 424, 589 (2004)

Jørgensen, J.K., Schöier, F.L., van Dishoeck, E.F.: Astron. Astrophys. 416, 603 (2004)

Jørgensen, J.K., Bourke, T.L., Myers, P.C., et al.: Astrophys. J. 632, 973 (2005)

Jørgensen, J.K., Harvey, P.M., Evans, N.J. II, et al.: Astrophys. J. 645, 1246 (2006)

Jørgensen, J.K., Bourke, T.L., Myers, P.C., et al.: Astrophys. J. 659, 479 (2007)

Kirk, J.M., Ward-Thompson, D., André, P.: Mon. Not. Roy. Astron. Soc. 375, 843 (2007)

Koerner, D.W., Sargent, A.I.: Astron. J. 109, 2138 (1995)

Krumholz, M.R., McKee, C.F., Klein, R.I.: Nature 438, 332 (2005)

Lada, C.J.: In: Lada, C.J., Kylafis, N.D. (eds.) The Origin of Stars and Planetary Systems, p. 143. Kluwer Academic, Dordrecht (1999)

Lahuis, F., van Dishoeck, E.F., Boogert, A.C.A., et al.: Astrophys. J. 636, L145 (2006)

Lin, S.-Y., Ohashi, N., Lim, J., et al.: Astrophys. J. 645, 1297 (2006)

Luhman, K.L., Joergens, V., Lada, C., et al.: In: Reipurth, B., Jewitt, D., Keil, K. (eds.) Protostars and Planets V, pp. 443-457. University of Arizona Press, Tucson (2007)

McKee, C.F., Tan, J.C.: Astrophys. J. 585, 850 (2003)

Mohanty, S., Jayawardhana, R., Natta, A., et al.: Astrophys. J. 609, L33 (2004)

Neufeld, D.A., Hollenbach, D.J.: Astrophys. J. 428, 170 (1994)

Piétu, V., Dutrey, A., Guilloteau, S.: Astron. Astrophys. 467, 163 (2007)

Qi, C., Kessler, J.E., Koerner, D.W., Sargent, A.I., Blake, G.A.: Astrophys. J. 597, 986 (2003)

Rebull, L.M., Stapelfeldt, K.R., Evans, N.J. II, et al.: Astrophys. J. Suppl. 171, 447 (2007)

Richer, J.S., Shepherd, D.S., Cabrit, S., Bachiller, R., Churchwell, E.: In: Mannings, V., Boss, A.P., Russell, S.S. (eds.) Protostars and Planets IV, p. 867. University of Arizona Press, Tucson (2000)

Shang, H., Li, Z.-Y., Hirano, N.: In: Reipurth, B., Jewitt, D., Keil, K. (eds.) Protostars and Planets V, pp. 261-276. University of Arizona Press, Tucson (2007)

Shepherd, D.S., Churchwell, E.: Astrophys. J. 457, 267 (1996)

Shepherd, D.S., Kurtz, S.E.: Astrophys. J. 523, 690 (1999)

Simon, R., Jackson, J.M., Rathborne, J.M., Chambers, E.T.: Astrophys. J. 639, 227 (2006)

Tafalla, M., Mardones, D., Myers, P.C., et al.: Astrophys. J. 504, 900 (1998)

Takahashi, S., Saito, M., Takakuwa, S., Kawabe, R.: Astrophys. J. 651, 933 (2006)

Testi, L., Natta, A., Oliva, E., et al.: Astrophys. J. 571, L155 (2002)

van der Tak, F.F.S., Caselli, P., Ceccarelli, C.: Astron. Astrophys. 439, 195 (2005)

van Dishoeck, E.F.: Proc. National Acad. Sci. 103, 12249 (2006)

Williams, J.P., Blitz, L., McKee, C.F.: In: Mannings, V., Boss, A.P., Russell, S.S. (eds.) Protostars and Planets IV, p. 97. University of Arizona Press, Tucson (2000)

Young, C.H., Jørgensen, J.K., Shirley, Y.L., et al.: Astrophys. J. Suppl. Ser. 154, 396 (2004) 